\title{
Salivary Cortisol and Binge Eating Disorder in Obese Women After Surgery for Morbid Obesity
}

\author{
Junilla K. Larsen • Bert van Ramshorst • \\ Lorenz J. P. van Doornen • Rinie Geenen
}

Published online: 14 March 2009

(C) The Author(s) 2009. This article is published with open access at Springerlink.com

\begin{abstract}
Background Binge eating episodes characterized by loss of control are hypothesized to be accompanied by changes in hypothalamic pituitary adrenal (HPA) axis functioning. Cortisol is an end product of this neuroendocrine stress system.

Purpose The aim of this study was to examine the cortisol levels and the awakening cortisol response (ACR) in obese persons showing binge eating after surgery for morbid obesity.

Method Sixteen obese women with binge eating disorder (BED) and 18 obese women without BED participated in the study. Means \pm SD: age $43 \pm 15$, body mass index $40 \pm 8$. Salivary cortisol, anthropometric assessments, and the eating disorder examination interview were taken.

Results Women with BED showed a significantly lower waist-to-hip ratio and cortisol levels during the day than women without BED, whereas the ACR did not differ.
\end{abstract}

J. K. Larsen · L. J. P. van Doornen · R. Geenen

Department of Clinical and Health Psychology,

Utrecht University,

Utrecht, The Netherlands

J. K. Larsen ( $\bowtie)$

Behavioural Science Institute, Radboud University Nijmegen,

P.O. Box 9104, 6500 HE Nijmegen, The Netherlands

e-mail: j.larsen@pwo.ru.nl

B. van Ramshorst

Department of Surgery, St. Antonius Hospital,

Nieuwegein, The Netherlands

R. Geenen

Department of Rheumatology and Clinical Immunology, University Medical Center Utrecht,

Utrecht, The Netherlands
Conclusion Our cross-sectional study in a small sample generates the hypothesis that neuroendocrine regulation differs between obese women with and without BED after obesity surgery. This finding needs replication in future studies that should also examine the causal direction of the observed association.

Keywords Salivary cortisol - Eating disorders · Obesity · Circadian rhythm . Cortisol awakening response .

Binge eating

\section{Introduction}

Obesity, defined as a body mass index (BMI) $\geq 30 \mathrm{~kg} / \mathrm{m}^{2}$, has become a major public health problem in industrialized countries. Part of obese individuals has binge eating disorder (BED), recurrent episodes of uncontrolled overeating, often involving extremely large amounts of foods, and feelings of depression, guilt, and disgust about the loss of control over eating and weight and shape appearance. Obese individuals with BED exhibit greater psychopathology and often do less well in weight loss treatment compared to obese individuals without BED [1].

The stress hormone cortisol may play a role in BED. Stress is a primary trigger for binge eating [2]. Cortisol is secreted by the adrenal cortex upon activation of the hypothalamus pituitary adrenal (HPA) axis in response to physiological or psychological stress, in particular when the individual is threatened with loss of control [3]; also, food intake increases cortisol levels [4]. Repeated HPA axis stimulation may lead to neurobiological changes that promote the compulsive nature of overeating [5]. Although stress, loss of control, and food intake are reasons for patients with BED to have elevated cortisol levels, the 
repeated and enduring activation of the adrenal cortex may ultimately result in relatively low cortisol levels [6-8].

Three previous studies that examined cortisol levels among obese women with and without BED were characterized by a single measurement of cortisol. One study found no differences in evening cortisol levels [9]. One study found no differences in morning cortisol levels [10], but another study found higher morning cortisol levels among obese women with BED, but no differences in daytime cortisol levels [11]. The salivary cortisol response to awakening has been described as a test of the susceptibility of the HPA axis to stress. The aim of the current study was to examine daytime salivary cortisol levels and the awakening cortisol response (ACR) among obese women with and without BED using cortisol assessment at two consecutive days at six time points. All women in our study had undergone laparoscopic adjustable gastric banding for severe obesity. Although binge eating will be more difficult for patients with a gastric band, it is still possible to eat large quantities of food that easily pass the gastric band, such as chocolate or ice cream.

\section{Method}

\section{Procedure}

Fifty females were selected for this study. They were recruited from a large cross-sectional survey study [12] from which the data were gathered 16 months before. Overweight women $(n=25)$ who met the criteria for severe binge eating, score $\geq 27$ on the binge eating scale (BES) [13], were matched to overweight women $(n=25)$ without binge eating problems (BES score $\leq 17$ ) on demographic characteristics (age, marital status, and educational level) and depressive symptoms. Forty-six women agreed to participate and were visited at home by a research assistant
(VC or CdL). During the visit, the clinical eating disorder examination (EDE) interview was administered to assess BED and eating psychopathology [14]. Height, weight, and the waist and hip circumferences were measured at the end of the interview. Two women with normal BMI's of $25 \mathrm{~kg} / \mathrm{m}^{2}$ were excluded from the study because they were not overweight. Nearly all participants were obese (BMI $\geq 30 \mathrm{~kg} / \mathrm{m}^{2}$ ); two women had BMIs of $29 \mathrm{~kg} / \mathrm{m}^{2}$. Hence, we decided to use the term "obese women" instead of "overweight women" in this article. Participants were given instructions on cortisol assessments for the next 2 days using a standardized protocol. Cortisol samples of 37 women were received (response rate $84 \%$ ). Cortisol assessments of three out of 37 participants were incomplete, leaving 34 evaluable women: 16 obese women with BED and 18 obese women without BED.

\section{Participants}

Table 1 provides an overview of demographic and weight data. Compared to obese women without BED, obese women with BED did not differ on demographic characteristics, medication use influencing the HPA system, and depression score. Also, the preoperative BMI did not differ between the groups. After the operation, women without BED had a significantly lower waist-to-hip ratio (WHR) compared to women with $\operatorname{BED}[t(30)=2.3, p<0.05]$; the BMI was marginally significantly lower among the nonBED participants $[t(32)=-1.8, p=0.08]$.

\section{Measurements}

Cortisol Assessment Saliva for cortisol assessments was collected with small cotton wool swabs (Salivette, Sarstedt AG \& Co., Numbrecht, Germany) in the real-life environment at six time points during two consecutive days. Saliva samples were collected at awakening, at 15 and $30 \mathrm{~min}$ after
Table 1 Characteristics of obese women with and without BED

a An education level of " 1 " reflects primary education; "2" lower secondary education; "3", " 4 ", and " 5 " secondary education; and " 6 " and " 7 " tertiary education

${ }^{b}$ Three women with BED and three women without BED used antihypertensive medication; none of the women used diabetes medication or other medication that could influence the HPA axis

\begin{tabular}{|c|c|c|c|c|}
\hline & \multicolumn{2}{|c|}{$\operatorname{BED}(n=16)$} & \multicolumn{2}{|c|}{ Non-BED $(n=18)$} \\
\hline & Mean & SD & Mean & SD \\
\hline Postoperative period (months) & 42.6 & 15.1 & 46.0 & 13.1 \\
\hline Age (years) & 38.4 & 7.6 & 42.7 & 6.5 \\
\hline Education level $^{\mathrm{a}}$ & 3.4 & 1.9 & 3.7 & 1.5 \\
\hline Preoperative BMI $\left(\mathrm{kg} / \mathrm{m}^{2}\right)$ & 46.6 & 6.3 & 46.1 & 5.5 \\
\hline Postoperative BMI $\left(\mathrm{kg} / \mathrm{m}^{2}\right)$ & 40.2 & 8.2 & 35.8 & 6.0 \\
\hline Postoperative waist-to-hip ratio & 0.85 & 0.09 & 0.92 & 0.08 \\
\hline Depressive symptoms (BDI) & 14.9 & 9.4 & 13.1 & 6.4 \\
\hline Use of oral contraceptives $(\%)$ & 38 & & 33 & \\
\hline Use of other medication influencing the HPA system ${ }^{\mathrm{b}}(\%)$ & 19 & & 17 & \\
\hline Marital status: percent single & 28 & & 31 & \\
\hline
\end{tabular}


awakening, and at 12:00 P.M., 3:00 P.M., and 8:00 P.M. Participants were instructed to rinse their mouth with water at each sampling point and to refrain from eating, drinking, smoking, or brushing their teeth in the $30 \mathrm{~min}$ before sampling. Participants stored the saliva samples in a refrigerator until, at the end of the 2 days, samples were sent to Utrecht University where they were kept frozen at $-20^{\circ} \mathrm{C}$ until analysis with a time-resolved immunoassay with fluorescence detection, DELFIA [15].

Anthropometric Assessment Height was measured without shoes, and body weight was determined to the nearest $0.5 \mathrm{~kg}$ using two identical weight balances. The BMI was calculated as the weight in kilograms divided by the square of height in meters. The waist and hip circumferences were measured, with the subjects standing, using a 1-cm-wide measuring tape, and WHR was calculated. According to the recommendation of the $\mathrm{WHO}$, the waist circumference was measured as the minimum value between the iliac crest and the lateral costal margin, whereas hip circumference was determined as the maximum value over the buttocks.

Eating Disorder Examination Interview Binge eating episodes and eating disorder psychopathology were assessed with the EDE interview [14]. Four subscales reflect eating disorder psychopathology: restraint, eating concern, shape concern, and weight concern. The items assessing eating disorder features for the four scales focus on the previous 28 days and are rated on a seven-point format (0 to 6) with higher scores reflecting greater severity or frequency. All interviews, except one, were audiotaped and reviewed by the two research assistants who did the interviews and a third judge (JKL) for the assessment of binge eating episodes. All judges were blinded to participants' BES scores that had been used to select the participants. BED was defined by the presence of a weekly average of at least one binge eating episode within the past 3 months [16]. There was a high correspondence between the judges concerning the presence of binge eating episodes with only one patient being classified differently.

\section{Statistical Analyses}

A common criterion used to decide on the normality of score distributions in small samples is that the skewness divided by the standard error of the skewness should be below standardized scores of 3.29 [17]. According to this criterion, all variables were normally distributed, except for the cortisol variable. There were five univariate cortisol outliers $(z>3.29)$ that were assigned a score that was one unit larger than the next most extreme score in the distribution [17]. The score distribution of the resulting cortisol values was not significantly skewed anymore. Therefore, it was possible to apply parametric statistics. The mean values of the cortisol samples over the two sampling days were used for analyses. Cronbach's alpha of the cortisol assessments over the 2 days was 0.71 . Repeated-measures analysis was used to examine overall differences in cortisol level over the six values between women with and without BED. As a measure of the ACR, the mean level (ACR level: 0, 15, and $30 \mathrm{~min}$ after awakening) and the increase of the cortisol measurements as a function of the time $(0,15$, and $30 \mathrm{~min})$ after awakening was calculated: the unstandardized regression coefficient of the regression equation was used (ACR slope). Analyses of variance were used to examine women with and without BED on the ACR and eating disorder pathology (EDE scores). All analyses were controlled for BMI as a covariate and were done with SPSS 14.0 for Windows.

\section{Results}

BED and Eating Disorder Psychopathology

Obese women with BED had significantly higher scores than obese women without BED on shape concern, $2.8 \pm 1.5$ versus $1.8 \pm 1.0, F(1,31)=4.5, p=0.04$, restraint $1.8 \pm 1.1$ versus $0.6 \pm 0.8, F(1,31)=9.0, p<0.01$, and eating concern,

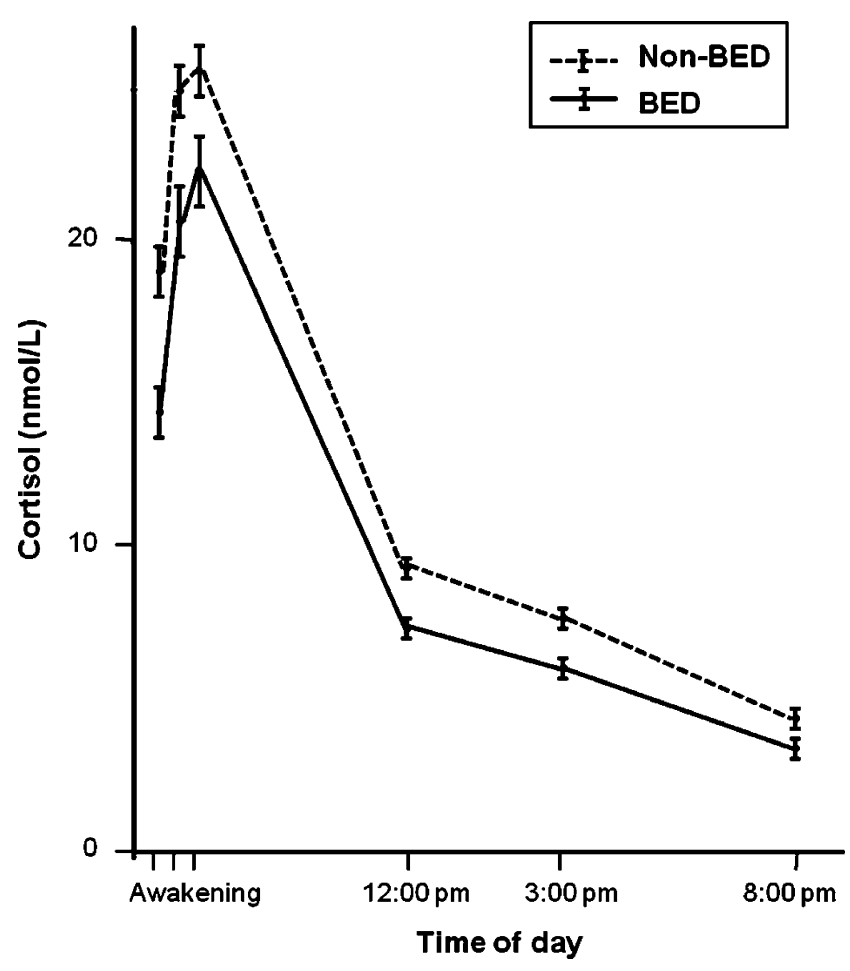

Fig. 1 Mean cortisol concentrations $( \pm \mathrm{SE})$ across the day of women with (solid line) and without (dashed line) BED 
$2.6 \pm 1.6$ versus $1.1 \pm 1.1, F(1,31)=8.2, p=0.007$, whereas they did not differ significantly on weight concern, $2.5 \pm 1.6$ versus $1.8 \pm 1.2, F(1,31)=1.3$, n.s. In terms of effect sizes, the differences on restraint, eating concern, and shape concern are large.

\section{BED and Cortisol}

Figure 1 displays the cortisol concentrations. Repeatedmeasures analysis showed that obese women with BED had significantly lower total cortisol levels during the day than obese women without BED, $F(1,31)=5.4, p=0.03$. This effect remained significant after additional adjustment for WHR or depressive symptoms. There were no significant differences on the ACR slope, $F(1,31)=1.4, p=0.25$, and ACR levels, $F(1,31)=3.5, p=0.07$. Comparison of the total cortisol level during the day using the area under the curve [18] yielded a large difference between women with $(9.0 \pm 2.4)$ and without (10.9 \pm 2.4 ) BED: the effect size $d$ was 0.8 [19].

\section{Discussion}

The present study examined the association between binge eating and salivary cortisol in obese women after bariatric surgery. Obese women with BED showed lower overall cortisol levels compared to obese women without BED, whereas the cortisol response to awakening was not related to BED. Before discussing this result, we reflect on the differences in eating psychopathology between obese women with and without BED.

Obese women with BED scored significantly higher on three out of four measures of eating disorder psychopathology compared to obese women without BED. The scores of our women with BED were rather similar to the scores of BED patients that have been previously reported [20]. This suggests that individuals with a gastric band who have BED are truly eating disordered patients.

Previous studies that examined cortisol levels among obese women with and without BED [9-11] were all characterized by a single measurement of cortisol. Our study was the first to examine the cortisol concentrations with repeated measurement across consecutive days. We observed that the overall cortisol level during the day was lower for obese women with BED than for obese women without BED. To be able to judge whether the levels of women with BED are decreased or the levels of women without BED are increased, future research should include reference values of a control population obtained under similar conditions and analyzed in single run. Our main finding indicates a difference in neuroendocrine regulation between obese women with and without BED after obesity surgery.
The WHR of our non-BED group was higher compared to the BED group. This finding is remarkable, particularly considering that the non-BED group had a trend towards a lower BMI. Our non-BED group had both higher WHR scores and cortisol levels than our BED group. Visceral obesity characterized by a high WHR is a main characteristic of the metabolic syndrome. Glucocorticoid-mediated visceral obesity induced by depression has been suggested $[21,22]$. Though our BED and non-BED groups were rather similar with respect to depressive symptoms, it may be that binge eating-related depression is different from nonbinge eating-related depression. Future prospective research should examine the hypothesis that higher cortisol levels may promote abdominal obesity [6] by focusing on the relation between the presence and absence of BED, depression, neuroendocrine regulation, and visceral obesity after obesity surgery.

A major limitation of our study is the small sample size. A moderate difference in awakening cortisol levels between the BED and non-BED group was not significant, but the power was enough to detect a large group difference in total cortisol levels across the day. Moreover, our study did not answer the question if the difference in cortisol values reflect a primary defect or a secondary reaction to long-lasting stress, e.g., of consuming large amounts of food, or another cause. Finally, our design did not allow the conclusion that obese women with BED had reduced cortisol levels or that obese women without BED had higher cortisol levels because we did not include a normal weight control group in our study.

In conclusion, the present study in a small sample generates the hypothesis that neuroendocrine regulation differs between obese women with and without BED after obesity surgery. This finding needs replication in future studies that should also examine the causal direction of the observed association.

Acknowledgements The authors would like to thank Vivian Corten and Christel de Lange for the help with data collection and Pierre Zelissen for his comments on an earlier draft of this article.

Open Access This article is distributed under the terms of the Creative Commons Attribution Noncommercial License which permits any noncommercial use, distribution, and reproduction in any medium, provided the original author(s) and source are credited.

\section{References}

1. Yanovski SZ. Binge eating disorder: current knowledge and future directions. Obes Res. 1993;1:306-24.

2. Levine MD, Marcus MD. Eating behavior following stress in women with and without bulimic symptoms. Annals Behav Med. 1997;19:132-8

3. Biondi M, Picardi A. Psychological stress and neuroendocrine function in humans: the last two decades of research. Psychother Psychosom. 1999;68:114-50. 
4. Rosmond R, Holm G, Bjorntorp P. Food-induced cortisol secretion in relation to anthropometric, metabolic and haemodynamic variables in men. International Journal of Obesity and Related Metabolic Disorders. 2000;24:416-22.

5. Adam TC, Epel ES. Stress, eating and the reward system. Physiol Behav. 2007;91:449-58.

6. Bjorntorp P, Rosmond R. Neuroendocrine abnormalities in visceral obesity. International Journal of Obesity and Related Metabolic Disorders. 2000;24(Suppl 2):S80-5.

7. Heim C, Ehlert U, Hellhammer DH. The potential role of hypocortisolism in the pathophysiology of stress-related bodily disorders. Psychoneuroendocrinology. 2000;25:1-35.

8. Raison CL, Miller AH. When not enough is too much: the role of insufficient glucocorticoid signaling in the pathophysiology of stress-related disorders. Am J Psychiatr. 2003;160:1554-65.

9. Coutinho WF, Moreira RO, Spagnol C, Appolinario JC. Does binge eating disorder alter cortisol secretion in obese women? Eat Behav. 2007;8:59-64.

10. Monteleone P, Luisi M, De Filippis G, Colurcio B, Genazzani AR, Maj M. Circulating levels of neuroactive steroids in patients with binge eating disorder: a comparison with nonobese healthy controls and nonbinge eating obese subjects. Int J Eat Disord. 2003;34:432-40.

11. Gluck ME, Geliebter A, Hung J, Yahav E. Cortisol, hunger, and desire to binge eat following a cold stress test in obese women with binge eating disorder. Psychosom Med. 2004;66:876-81.

12. Larsen JK, Geenen R, van Ramshorst B, Brand N, de Wit P, Stroebe W, et al. Psychosocial functioning before and after laparoscopic adjustable gastric banding: a cross-sectional study. Obes Surg. 2003;13:629-36.

13. Gormally J, Black S, Daston S, Rardin D. The assessment of binge eating severity among obese persons. Addict Behav. 1982;7:47-55.
14. Jansen A. Interview ter vaststelling van de specifieke psychopathologie van eetstoornissen [Eating Disorder Examination: Interview to determine the specific psychopathology of eating disorder]. Lisse: Swets en Zeitlinger; 2000.

15. Dressendorfer RA, Kirschbaum C, Rohde W, Stahl F, Strasburger CJ. Synthesis of a cortisol-biotin conjugate and evaluation as a tracer in an immunoassay for salivary cortisol measurement. J Steroid Biochem Mol Biol. 1992;43:683-92.

16. Hsu LK, Mulliken B, McDonagh B, Krupa Das S, Rand W, Fairburn $\mathrm{CG}$, et al. Binge eating disorder in extreme obesity. International Journal of Obesity and Related Metabolic Disorders. 2002;26:1398-403.

17. Tabachnick BG, Fidell LS. Using multivariate statistics. Boston, MA: Allyn and Bacon; 2001.

18. Altman DG. Practical statistics for medical research. London: Chapman \& Hall; 1991.

19. Cohen J. Statistical power analysis for the behavioral sciences. 2nd ed. Hillsdale, NJ: Lawrence Erlbaum Associates; 1988.

20. Hrabosky JI, Masheb RM, White MA, Grilo CM. Overvaluation of shape and weight in binge eating disorder. J Consult Clin Psychol. 2007;75:175-80.

21. Chrousos GP. The role of stress and the hypothalamic-pituitaryadrenal axis in the pathogenesis of the metabolic syndrome: neuro-endocrine and target tissue-related causes. International Journal of Obesity and Related Metabolic Disorders. 2000;24 (Suppl 2):S50-5.

22. Vogelzangs N, Suthers K, Ferrucci L, Simonsick M, Ble A, Schrager M, et al. Hypercortisolemic depression is associated with the metabolic syndrome in late-life. Psychoneuroendocrinology. 2007;32:151-9. 